\title{
Molecular Docking and Molecular Dynamics Studies of SARS-CoV-2 Inhibitors: Crocin, Digitoxigenin, Beta- Eudesmol and Favipiravir: Comparative Study
}

\author{
José R. Mora ${ }^{1 \mathbb{D}}$, Sebastián A. Cuesta ${ }^{1 \mathbb{D}}$, Assia Belhassan ${ }^{2} \mathbb{D}$, G. Salgado Morán ${ }^{3 \mathbb{D}}$, Tahar \\ Lakhlifi $^{2}$ (D), Mohammed Bouachrine ${ }^{2(D)}$, Carlos Peña F. ${ }^{4}$ (D), Lorena Gerli C. ${ }^{\text {(D) }}$ Luis H. Mendoza- \\ Huizar 6,*(D)
}

1 Computational and Theoretical Chemistry Group (QCT-USFQ), Department of Chemical Engineering, San Francisco de Quito University, Diego de Robles y Vía Interoceánica, Quito 170901

2 Molecular Chemistry and Natural Substances Laboratory, Faculty of Science, Moulay Ismail University of Meknes, Morocco

3 Technische Universität Dresden Theoretische Chemie Ausseruniversitaerer Forscher, 01062, Dresden, Deutschland

4 Institute of Applied Chemical Sciences, Faculty of Engineering, Autonomous University of Chile, Av. Alemania 01090, 4810101, Temuco, Chile

5 Department of Environmental Chemistry, Faculty of Sciences, Catholic University of the Most Holy Conception, Concepción, Chile

6 Autonomous University of Hidalgo State. Academic Area of Chemistry. Mineral de la Reforma, Hidalgo, México

* Correspondence: hhuizar@uaeh.edu.mx (L.H.M.-H.);

Scopus Author ID 6507875400

Received: 2.07.2021; Revised: 28.08.2021; Accepted: 3.09.2021; Published: 20.10.2021

\begin{abstract}
In this study, Crocin, Digitoxigenin, Beta-Eudesmol, and Favipiravir were docked in the active site of SARS-CoV-2 main protease (PDB code: 6LU7). The docking study was followed by Molecular Dynamics simulation. The result indicates that Crocin and Digitoxigenin are the structures with the best affinity in the studied enzyme's binding site. Still, Molecular Dynamics simulation showed that Digitoxigenin is the molecule that fits better in the active site of the main protease. Therefore, this molecule could have a more potent antiviral treatment of COVID-19 than the other three studied compounds.
\end{abstract}

Keywords: Beta-Eudesmol; Crocin; Digitoxigenin; Favipiravir; SARS-CoV-2.

(C) 2021 by the authors. This article is an open-access article distributed under the terms and conditions of the Creative Commons Attribution (CC BY) license (https://creativecommons.org/licenses/by/4.0/).

\section{Introduction}

SARS-CoV-2 is a new type of coronavirus that causes COVID-19 disease; the first infection reported appeared on November 17, 2019, in Wuhan, China [1-4], and the symptoms caused by the symptoms of this virus have made evident the urgent need for new drugs to attack this disease. Due to the long time required to produce new drugs, molecules from natural extracts are emerging as a promising option for targeting new diseases and developing drugs with specific activity $[5,6]$. Nowadays, there are no proper antiviral therapies to treat patients with COVID-19. Therefore, to search for active and safe antiviral agents with broad-spectrum activity against this emerging and potentially fatal infection. In this sense, Crocin, Digitoxigenin, Beta-Eudesmol are natural molecules that have been proposed as potential inhibitors of SARS-CoV-2 main protease because they have significant antiviral power and according to experiment research [5]. For example, Crocin can inhibit the replication of the Herpes simplex virus (HSV) [7]. Furthermore, Digitoxigenin derivatives are used as antiviral 
and anti-cancer inhibitors [8], while Beta-Eudesmol has significant antibacterial and antiviral power [9]. Additionally, several works reported in the literature have suggested these three compounds to treat the coronavirus infection caused by SARS-CoV-2 [10,11].

On the other hand, T-705-4-ribofuranosyl-5-triphosphate (T-705RTP), also known as Favipiravir, is a chemical compound used against different types of RNA viruses [12]. This pyrazinamide derivative has been shown to be active on some types of viruses such as influenza, West Nile, yellow fever, and other types of arenaviruses, alphaviruses, bunyaviruses, and flaviviruses [13]. Also, it has been used experimentally to treat infection by the Ebola virus in humans [14]. Here, it is interesting to mention that the mechanism of action of favipiravir implies that it is being absorbed into cells and transformed into favipiravir ribofuranosyl phosphate due to the action of enzymes present in the host cell. This substance selectively inhibits the RNA polymerase of RNA viruses, which is essential for virus replication, without apparently producing toxicity to mammalian cells[13]. However, its safety and efficacy in humans are unknown in detail, as it is still experimental. China has completed the clinical investigation of the antiviral drug Favipiravir, which has shown its efficacy against the COVID-19[15], as confirmed by the country's government and reported by the Xinhua news agency[16]. This drug was approved for clinical use in Japan in 2014 [17]. Thus, according to the above mentioned, the study of interactions between Crocin, Digitoxigenin, Beta-Eudesmol, and Favipiravir against SARS-CoV-2 main protease can be useful to understand their action mechanism.

In the last two decades, computer-aided drug design, which employs computational approaches to drug discovery, development, and analysis, has positively impacted drug discovery and development [18-20]. It is well accepted that the study of drug binding to the receptor protein at the molecular level is of utmost importance to identify the pharmacokinetic profile of drugs [21]. In this sense, molecular docking is essential to dock the 3D structure of a molecule (ligand) into the binding site of a protein receptor to identify their molecular interactions [22]. The docking process involves two main and independent steps: the first one corresponds to the generation of the correct pose (conformation and orientation) of the ligands within the receptor binding site (protein), while the second one is related to the evaluation of the binding affinity [22]. Molecular docking has contributed significantly to reducing both the experimental work, the associated costs, and the time required to design a new drug[20].

Thus, in this investigation, molecular docking of Crocin, Digitoxigenin, BetaEudesmol, and Favipiravir into the active site of SARS-CoV-2 main protease (Code PDB: 6LU7), was used to predict the mode of binding between these molecules and their potential target (SARS-CoV-2 main protease), and to determine the affinity of these molecules in the binding site. Also, the molecular docking study was followed by Molecular Dynamic simulations to study the stability of complexes formed.

\section{Materials and Methods}

\subsection{Data set.}

The chemical compounds reported in Table 1 (Crocin, Digitoxigenin, Beta-Eudesmol, and Favipiravir) were tested as potential inhibitors of SARS-CoV-2 mean protease. 
Table 1. Chemical structures of Crocin, Digitoxigenin, Beta-Eudesmol and Favipiravir.

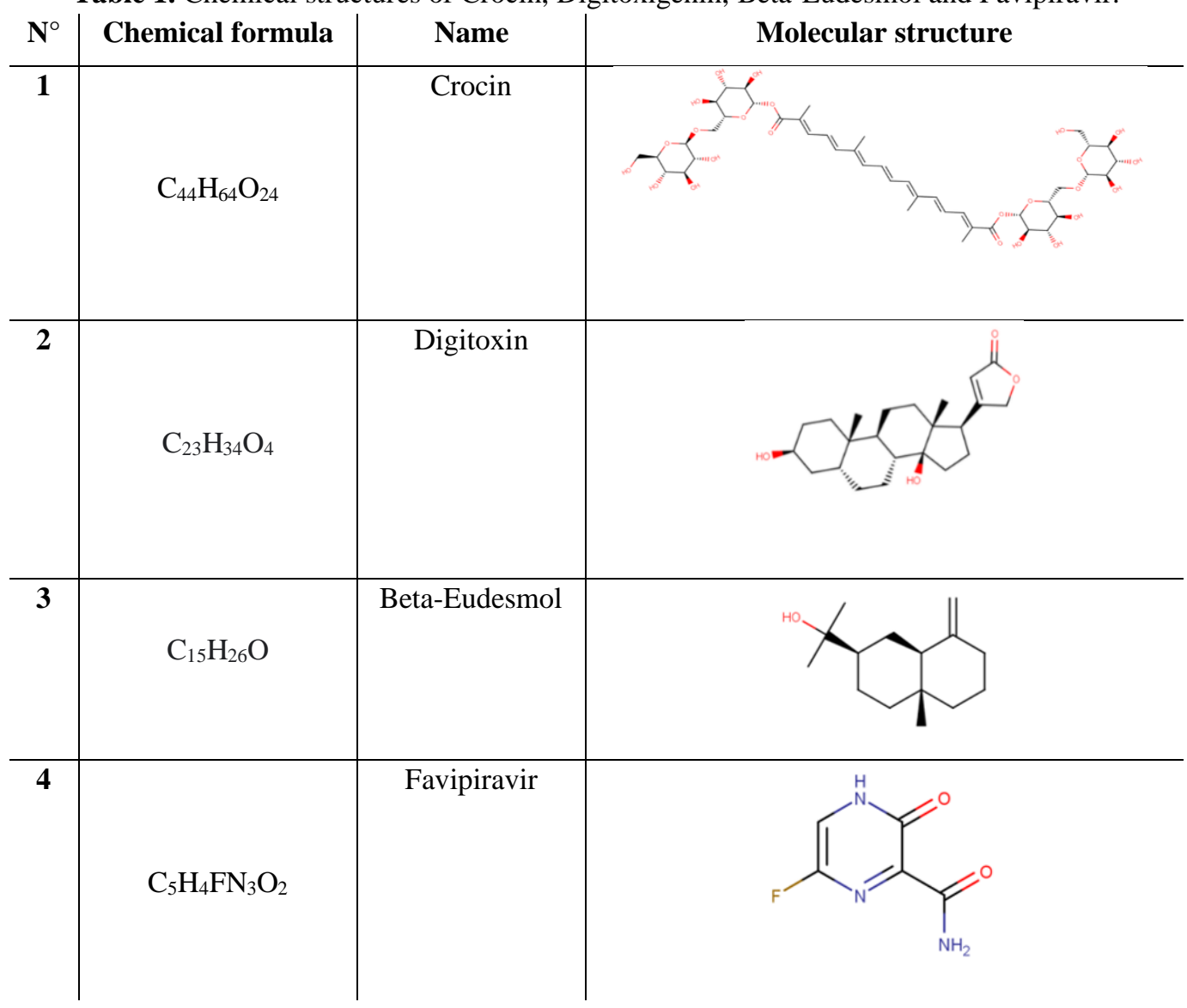

2.2. Molecular docking.

Molecular docking was performed with two programs: Autodock vina [23] and Autodock tools 1.5.6 [24]; to determine the binding affinity and predict the intermolecular interactions of molecules in the studied binding pocket of the studied enzyme (pdb code 6LU7) [25]. The crystallographic structure of the SARS-CoV-2 main protease used in this study is characterized by: Code: 6LU7 and Resolution of $2.16 \AA$ A. Discovery Studio Modeling Environment Release 2017 program [26] was used to detect binding site location and the center of the active site $(x=-10.782, y=15.787$ and $z=71.277)$ [27]. The grid size was set at $20 \times 34 \times 20$ xyz points with a grid spacing of $1 \AA$ and was generated by using the co-crystallized (N3) inhibitor as the center for docking [27,28].

\subsection{Molecular dynamic simulations.}

Molecular dynamic (MD) simulations were performed in the four studied molecules: Crocin, Digitoxigenin, Beta-eusdesmol, and in the experimental antiviral drug Favipiravir. The simulation was done to analyze the stability of the ligand-enzyme complex where different insights on the interaction can be obtained. To run the MD simulations, the enzyme prepared for the docking was employed. For the ligands, the pose with the best docking score was chosen as starting position. The whole system was solvated using a water cube, and sodium or chlorine atoms were added to neutralize it. All calculations were performed using Gromacs 2019 software [29] and CHARMM 2017 force field. Before the simulation, the system was relaxed and then equilibrated for a period of 100 past constant temperature and pressure ( 1 bar and 300 $\mathrm{K})$. The dynamic molecular production was then run for $10 \mathrm{~ns}$. 
Finally, binding free energy calculations were performed using the Molecular Mechanics Poisson-Boltzmann Surface Area (MM-PBSA) technique incorporated in the g_mmpbsa tool [30].

\section{Results and Discussion}

\subsection{Docking results.}

Molecular docking was performed to find interactions and the binding affinity of studied molecules in studied enzymes. Four different molecules (Crocin, Digitoxigenin, BetaEudesmol, and Favipiravir) have been evaluated for their affinity against the SARS-CoV-2 main protease. The results are presented in Table 2.

Table 2. Docking score results: Affinity of the best conformation in the binding pocket of SARS-CoV-2 main

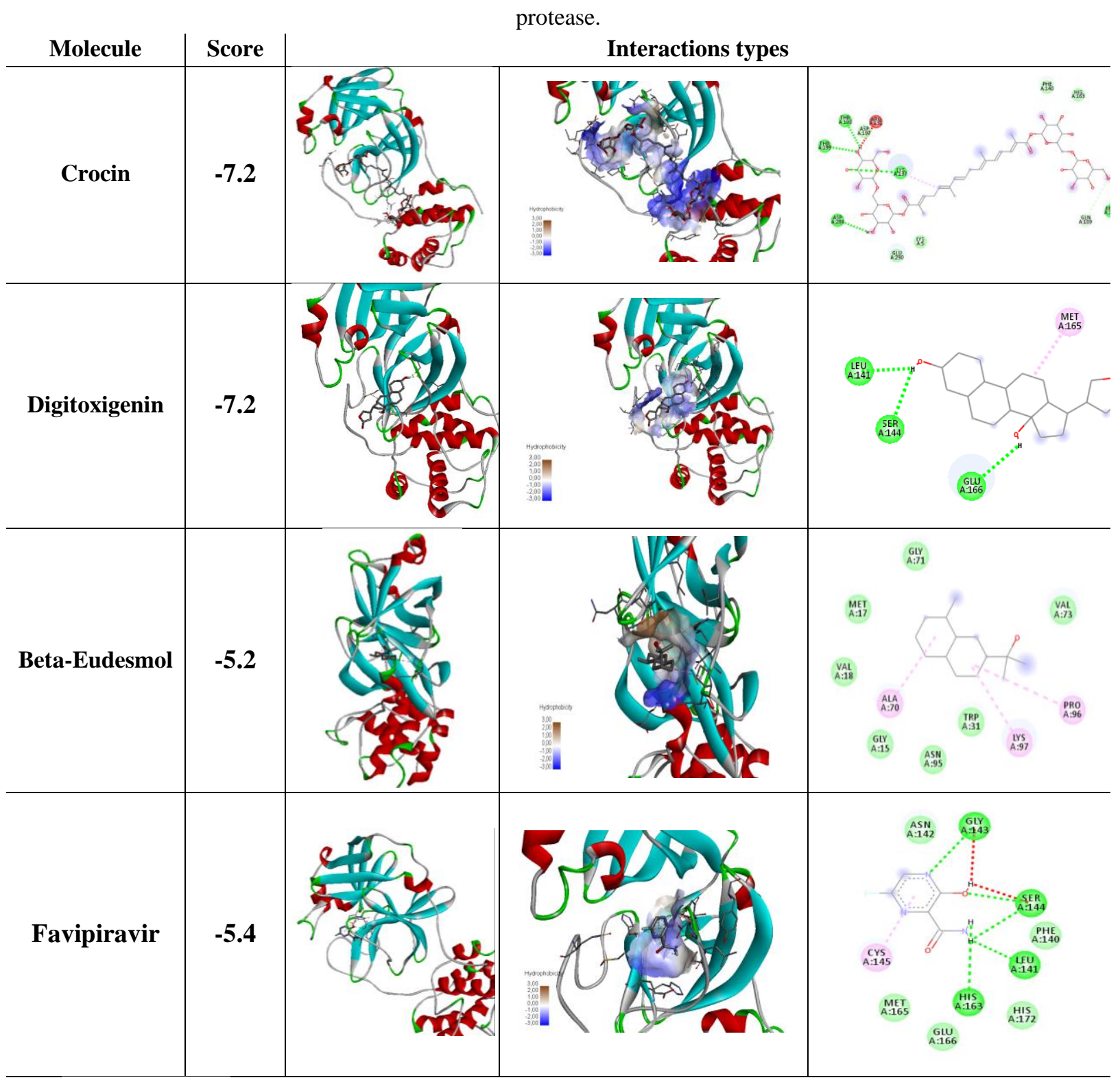

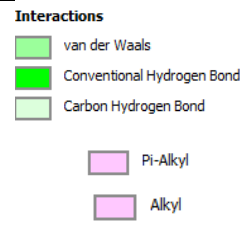


The best energies of interaction with SARS-CoV-2 main protease are observed for Crocin and Digitoxigenin (Table 2); these compounds could have more inhibitory potential SARS-CoV-2 main protease in comparison to the other studied compounds (Beta-Eudesmol and Favipiravir). Thus, the inhibition of this protein could induce the inhibition of viral replication.

\subsection{Molecular dynamics simulation.}

In a molecular dynamic simulation, the enzyme's flexibility and the interaction between the ligand, the enzyme, and water molecules over time are considered. In Figure 1, the potential energy of the different systems is shown. It can be seen that for all models, the potential energy in all cases reaches a plateau before 1200 ps showing that for most of the simulation, the system is at its lowest potential energy.

Beta-eusdesmol

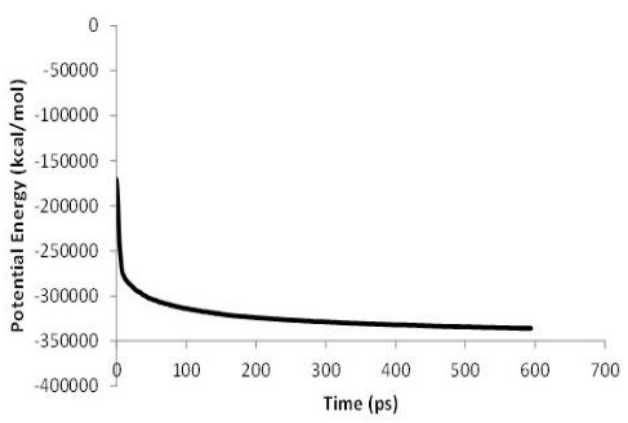

Digitoxigenin

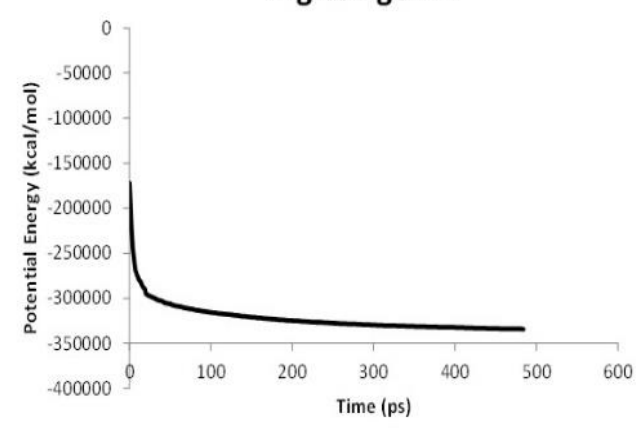

Crocin

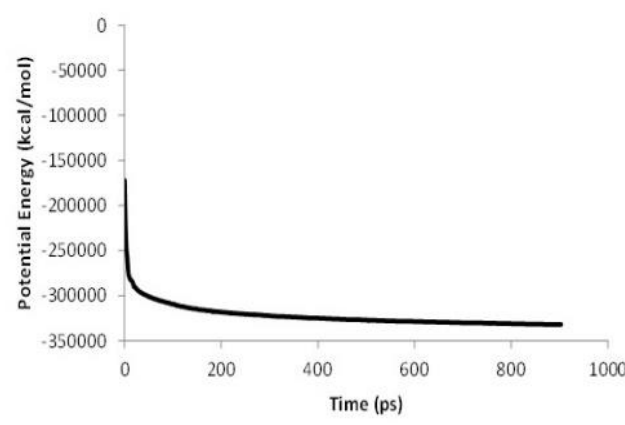

Favipiravir

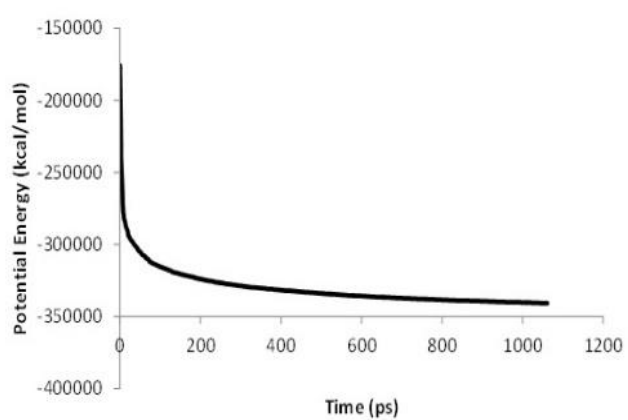

Figure 1. Potential energy of the MD simulations of different compounds with SARS-CoV-2 Main protease.

Figure 2 shows the root-mean-square deviation for the ligand and the system. As expected, the ligand presents higher RMSD values because smaller molecules tend to move and vibrate more than bigger enzymatic systems.

The RMSD value for the ligand-Main protease complex is around $0.2 \mathrm{~nm}$ in the four studied systems. For the ligands, Crocin is the one that presents higher RMSD values, followed by Digitoxigenin, Favipiravir, and finally Beta-eusdesmol. Digitoxigenin RMSD values are higher $(\sim 0.9 \mathrm{~nm})$ in the first $3 \mathrm{~ns}$ of the simulation, meaning a great molecule movement. Then, in the last $7 \mathrm{~ns}$, the molecule stabilizes, and the RMSD values lower up to $0.6 \mathrm{~nm}$. Favipiravir behavior is the opposite, where the RMSD value is around $0.4 \mathrm{~nm}$ in the first part of the simulation, raising to $1 \mathrm{mn}$ in the last half of the simulation. For Beta-eusdesmol, it maintains its RMSD value around 0.6 throughout the whole simulation. 
Beta-eusdesmol

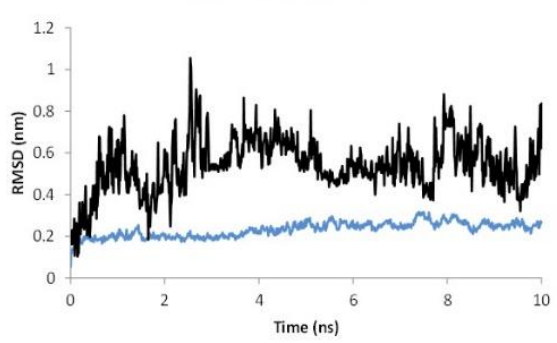

Digitoxigenin

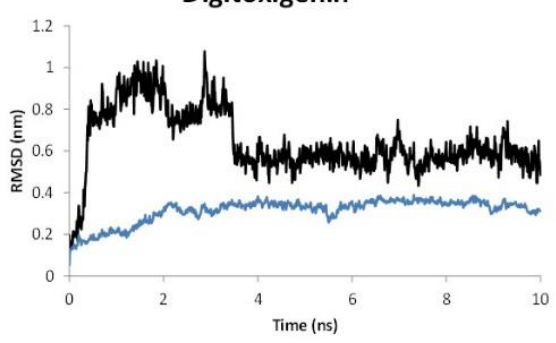

Crocin

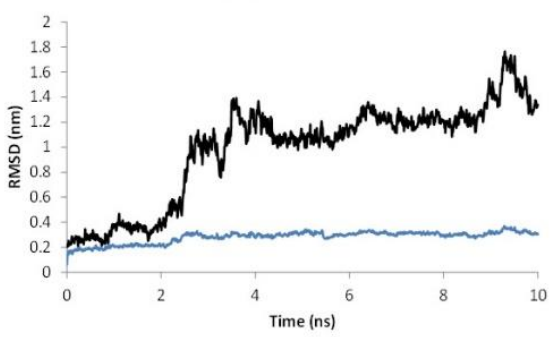

Favipiravir

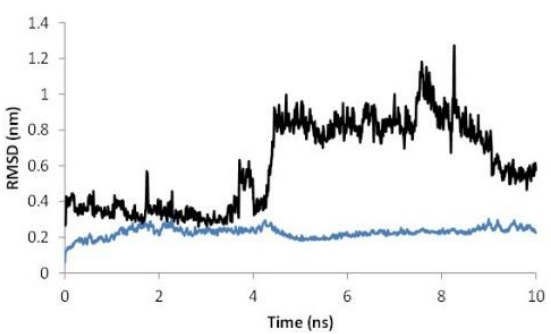

Figure 2. RMSD of the ligand (black) and the ligand-enzyme complex (blue) vs. simulation time.

Then, Coulomb and Lennard-Jones' interaction energies were studied (Figure 3). At the end of the simulation, Crocin presents the lowest Coulomb and Lennard-Jones values indicating a strong interaction between the ligand and the main protease. Beta-eusdesmol and Digitoxigenin present Coulomb energy values between 0 and $-10 \mathrm{kcal} / \mathrm{mol}$, while antiviral Favipiravir has values $-10 \mathrm{kcal} / \mathrm{mol}$ lower. Digitoxigenin values for the Lennard-Jones energy are the second lowest, followed by Favipiravir and Beta-eusdesmol.

Analyzing hydrogen bond formation during the simulation (Figure 4), it was found that Crocin is the molecule forming the most number of hydrogen bonds, followed by Favipiravir, Digitoxigenin, and Beta-eusdesmol. At the beginning of the simulation, Crocin forms around 9 hydrogen bonds, reducing them to four at the end of the simulation.

Beta-eusdesmol

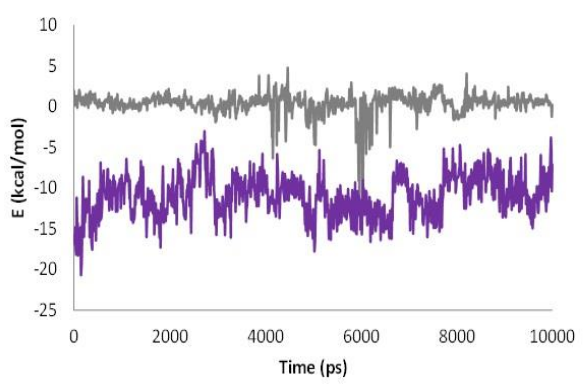

Digitoxigenin

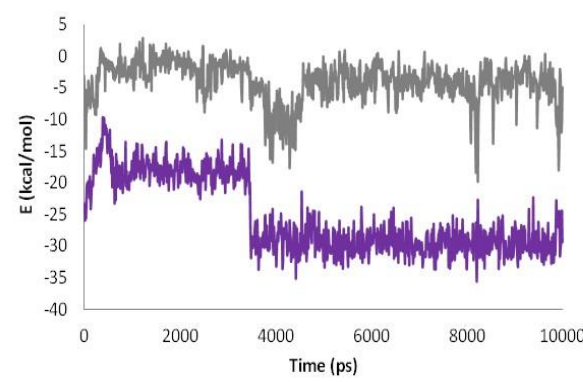

Crocin

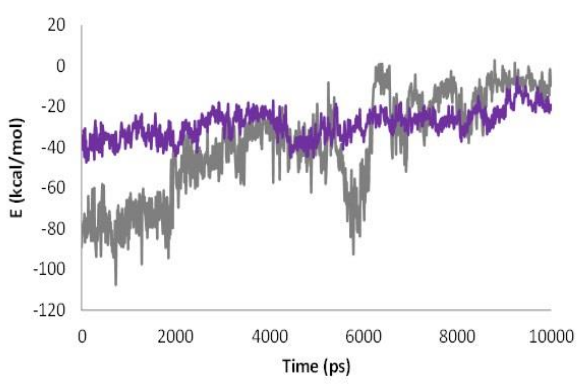

Favipiravir

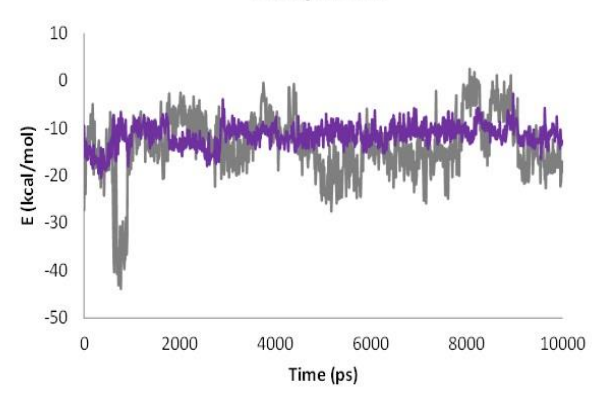

Figure 3. Coulomb (grey) and Lennard-Jones (purple) interaction energy during the simulation. 
Favipiravir forms on average 3 hydrogen bonds throughout the simulation. Digitoxigenin forms only one hydrogen bond during the $10 \mathrm{~ns}$, while Beta-eusdesmol does not form any hydrogen bond in most simulation parts. Bond formation agrees with interaction energy; as more hydrogen bonds are formed, a better and stronger interaction occurs between the ligand and the enzyme.

Beta-eusdesmol

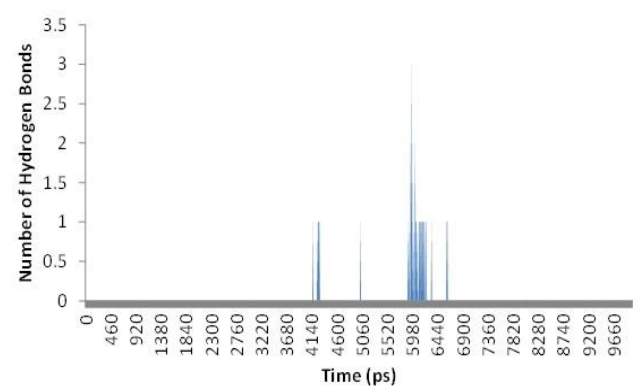

Digitoxigenin

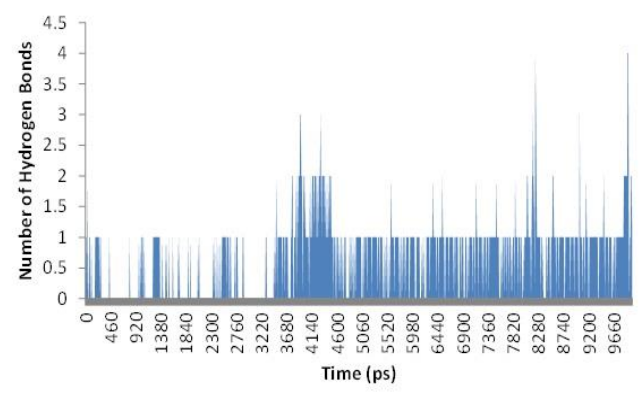

Crocin

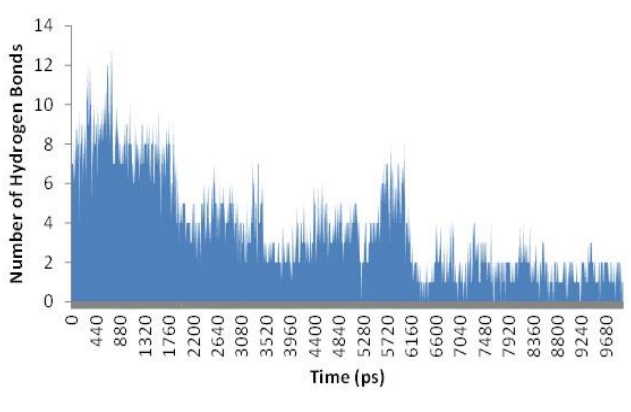

Favipiravir

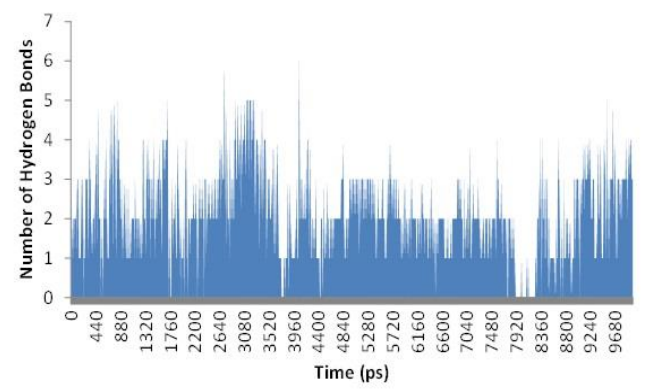

Figure 4. Number of hydrogen bonds vs. simulation time.

The initial and final poses of the simulation were compared for all four compounds, and the final pose was studied more in detail (Figure 5). For Beta-eusdesmol, both structures are outside and far from the active site of the main protease. Between the beginning and the end of the simulation, the molecule moves $77 \mathrm{~nm}$ keeping a superficial interaction with the enzyme. During the 10 ns simulation, Crocin leaves the enzyme's active site and is located in a long superficial area next to the pocket. Digitoxigenin and Favipiravir stay in the active site throughout the simulation time.

While Digitoxigenin moves inside the active site, fitting in a better way deeper in the pocket, the antiviral Favipiravir accommodates on the other end of the active site. 2D interaction plots show Beta-eusdesmol interacts with 3 residues, Crocin with 5, Digitoxigenin with 7, and Favipiravir with 6 residues.

This bears out that Beta-eusdesmol is the molecule that interacts less with the enzyme agreeing with the data obtained throughout the simulation and its location far from the active site. Crocin interactions occur only at one side of the molecule, leaving most of it without any interactions. Digitoxigenin and Favipiravir, as they are inside the pocket, interactions occur all around both molecules, which do not happen with Beta-eusdesmol that presents a superficial interaction only at one side. 

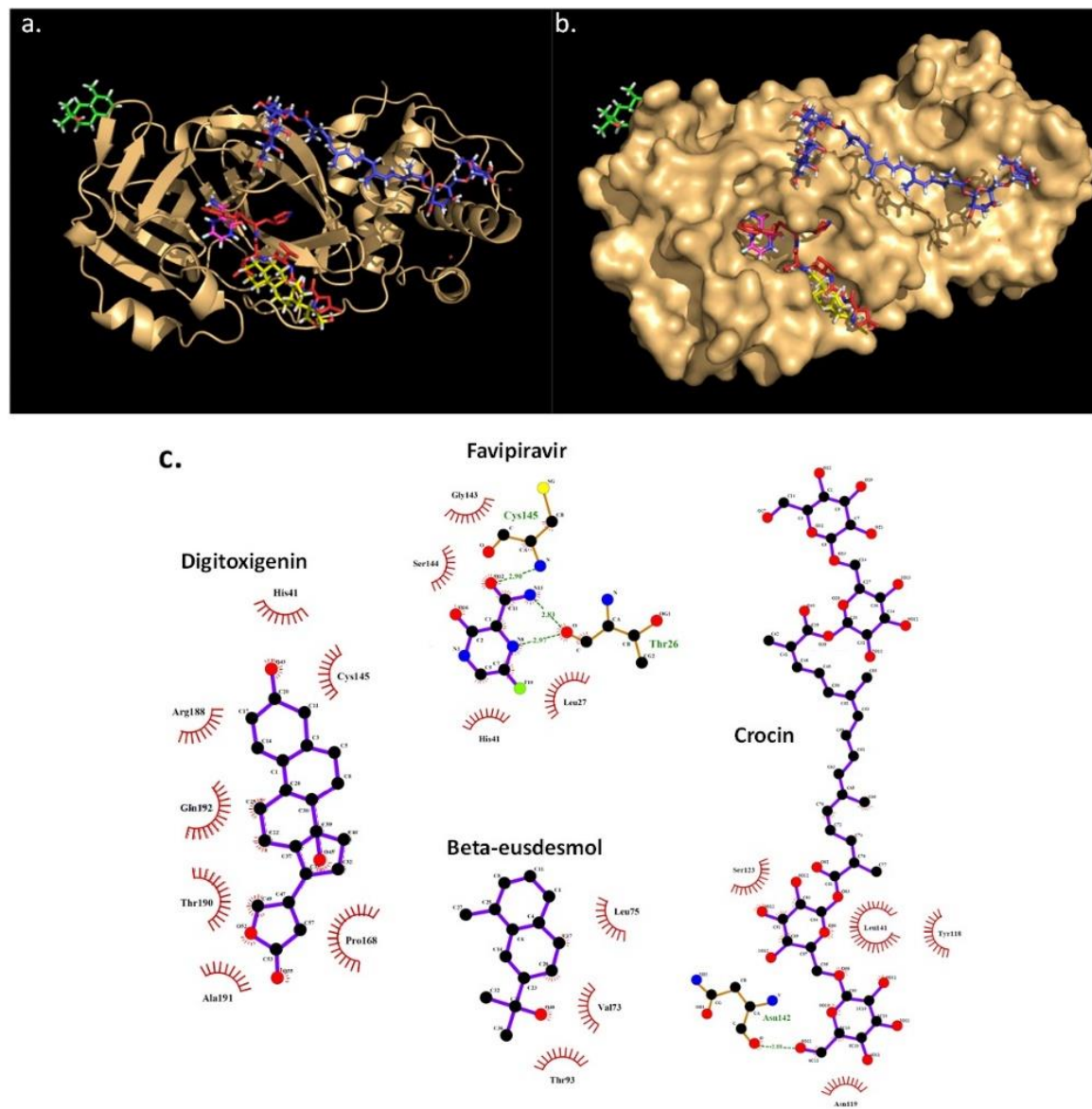

Figure 5. SARS-CoV-2 main protease (orange) interaction with Beta-eusdesmol (green), Crocin (blue), Digitoxigenin (yellow), Favipiravir (pink), and known inhibitor N3 (red): (a) cartoon representation; (b) surface representation; (c) $2 \mathrm{D}$ representation.

The total binding energy and their contributions were calculated and are presented in Table 3.

Table 3. Total binding energy and their contributions for four systems formed.

\begin{tabular}{c|c|c|c|c} 
Compound & $\begin{array}{c}\text { Van der Waals } \\
(\mathbf{k c a l} / \mathbf{m o l})\end{array}$ & $\begin{array}{c}\text { Electrostatics } \\
(\mathbf{k c a l} / \mathbf{m o l})\end{array}$ & $\begin{array}{c}\text { SASA } \\
(\mathbf{k c a l} / \mathbf{m o l})\end{array}$ & $\begin{array}{c}\text { Total Binding energy } \\
(\mathbf{k c a l} / \mathbf{m o l})\end{array}$ \\
\hline Crocin & -28.0 & -13.8 & -4.3 & -1.1 \\
\hline Digitoxigenin & -31.5 & -3.5 & -3.5 & -16.1 \\
\hline Beta-eusdesmol & -12.8 & -1.1 & -2.0 & -9.9 \\
\hline Favipiravir & -11.6 & -6.5 & -1.6 & -5.1
\end{tabular}

Table 3 shows that Digitoxigenin is the compound lowest binding energy. This was expected as it is the molecule that fits better in the active site of the main protease. Crocin presents the highest binding energy meaning an unfavorable interaction between this compound and the enzyme. This suggests that only Digitoxigenin may be able to exert an antiCOVID-19 effect through the main protease inhibition mechanisms of SARS-CoV-2.

\section{Conclusions}

In this study, we have performed a docking study of Crocin, Digitoxigenin, BetaEudesmol, and Favipiravir in the active site of SARS-CoV-2 main protease. The result indicates that Crocin and Digitoxigenin are the structures with the best affinity in the enzyme's binding site; MD simulation showed that Digitoxigenin is the molecule that fits better in the active site of the main protease. Crocin presents the highest binding energy meaning an 
unfavorable interaction between this compound and the enzyme. This result suggests that only Digitoxigenin may be able to exert an anti-COVID-19 effect through the main protease inhibition mechanisms of SARS- CoV-2.

\section{Funding}

The authors gratefully acknowledge the financial support of Direction of research UCSC, Project DIREG 03/2020. LHMH gratefully acknowledges financial support from the Universidad Autónoma del Estado de Hidalgo.

\section{Acknowledgments}

The authors are grateful for the administrative support provided by Universidad San Francisco de Quito, Universidad Moulay Ismail de Meknes, Technische Universität Dresden, Universidad Autónoma de Chile, Universidad Católica de la Santísima Concepción, Universidad Autónoma del Estado de Hidalgo, for the realization of this work.

\section{Conflicts of Interest}

The authors declare no conflict of interest.

\section{References}

1. Elfiky, A.A. Anti-HCV, nucleotide inhibitors, repurposing against COVID-19. Life Sciences 2020, 248, 117477, https://doi.org/10.1016/j.lfs.2020.117477.

2. Wu, C.; Liu, Y.; Yang, Y.; Zhang, P.; Zhong, W.; Wang, Y.; Wang, Q.; Xu, Y.; Li, M.; Li, X.; et al. Analysis of therapeutic targets for SARS-CoV-2 and discovery of potential drugs by computational methods. Acta Pharmaceutica Sinica B 2020, 10, 766-788, https://doi.org/10.1016/j.apsb.2020.02.008.

3. Singh, A.K.; Singh, A.; Shaikh, A.; Singh, R.; Misra, A. Chloroquine and hydroxychloroquine in the treatment of COVID-19 with or without diabetes: A systematic search and a narrative review with a special reference to India and other developing countries. Diabetes Metab Syndr 2020, 14, 241-246, http://doi.org/10.1016/j.dsx.2020.03.011.

4. Kang, D.; Choi, H.; Kim, J.H.; Choi, J. Spatial epidemic dynamics of the COVID-19 outbreak in China. Int. J. Infect. Dis. 2020, 94, 96-102, http://doi.org/10.1016/j.ijid.2020.03.076.

5. Aanouz, I.; Belhassan, A.; El-Khatabi, K.; Lakhlifi, T.; El-ldrissi, M.; Bouachrine, M. Moroccan Medicinal plants as inhibitors against SARS-CoV-2 main protease: Computational investigations. J. Biomol. Struct. Dyn. 2020, 39, 2971-2979, http://doi.org/10.1080/07391102.2020.1758790.

6. Sokolova, A.S.; Yarovaya, O.I.; Korchagina, D. V.; Zarubaev, V. V.; Tretiak, T.S.; Anfimov, P.M.; Kiselev, O.I.; Salakhutdinov, N.F. Camphor-based symmetric diimines as inhibitors of influenza virus reproduction. Bioorganic Med. Chem. 2014, 22, 2141-2148, http://doi.org/10.1016/j.bmc.2014.02.038.

7. Soleymani, S.; Zabihollahi, R.; Shahbazi, S.; Bolhassani, A. Antiviral Effects of Saffron and its Major Ingredients. Curr. Drug Deliv 2018, 15, 698-704, http://doi.org/10.2174/1567201814666171129210654.

8. Boff, L.; Munkert, J.; Ottoni, F.M.; Zanchett Schneider, N.F.; Ramos, G.S.; Kreis, W.; Fernandes de Andrade, S.; Dias de Souza Filho, J.; Braga, F.C.; Alves, R.J.; Maia de Pádua, R.; Oliveira Simões, C.M. Potential anti-herpes and cytotoxic action of novel semisynthetic digitoxigenin-derivatives. Eur J Med Chem 2019, 167, 546-561, http://doi.org/10.1016/j.ejmech.2019.01.076.

9. Schnitzler, P.; Astani, A.; Reichling, J. Screening for antiviral activities of isolated compounds from essential oils. Evidence-based Complement. Altern. Med. 2011, http://doi.org/10.1093/ecam/nep187.

10. Ghosh, R.; Chakraborty, A.; Biswas, A.; Chowdhuri, S. Evaluation of green tea polyphenols as novel corona virus (SARS CoV-2) main protease (Mpro) inhibitors-an in silico docking and molecular dynamics simulation study. J. Biomol. Struct. Dyn. 2020, http://doi.org/10.1080/07391102.2020.1779818.

11. Giguet-Valard, A.-G.; Raguette, K.; Morin, S.; Bellance, R.; Ravin, J.S. Gossypetin Derivatives are also Putative Inhibitors of SARS-COV 2: Results of a Computational Study. J. Biomed. Res. Environ. Sci. 2020, 1, 201-212, http://doi.org/10.37871/jbres1144. 
12. Naesens, L.; Guddat, L.W.; Keough, D.T.; Van Kuilenburg, A.B.P.; Meijer, J.; Vande Voorde, J.; Balzarini, J. Role of human hypoxanthine guanine phosphoribosyltransferase in activation of the antiviral agent T-705 (favipiravir). Mol. Pharmacol. 2013, 84, 615-629, http://doi.org/10.1124/mol.113.087247.

13. Furuta, Y.; Komeno, T.; Nakamura, T. Favipiravir (T-705), a broad spectrum inhibitor of viral RNA polymerase. Proc Jpn Acad Ser B Phys Biol Sci 2017, 93, 449-463, http://doi.org/10.2183/pjab.93.027.

14. Oestereich, L.; Lüdtke, A.; Wurr, S.; Rieger, T.; Muñoz-Fontela, C.; Günther, S. Successful treatment of advanced Ebola virus infection with T-705 (favipiravir) in a small animal model. Antiviral Res. 2014, 105, 17-21, http://doi.org/10.1016/j.antiviral.2014.02.014.

15. Costanzo, M.; De Giglio, M.A.R.; Roviello, G.N. SARS-CoV-2: Recent Reports on Antiviral Therapies Based on Lopinavir/Ritonavir, Darunavir/Umifenovir, Hydroxychloroquine, Remdesivir, Favipiravir and other Drugs for the Treatment of the New Coronavirus. Curr. Med. Chem. 2020, 27, 4536-4541, http://doi.org/10.2174/0929867327666200416131117.

16. Agrawal, U.; Raju, R.; Udwadia, Z.F. Favipiravir: A new and emerging antiviral option in COVID-19. Med J Armed Forces India 2020, 76, 370-376, http://doi.org/10.1016/j.mjafi.2020.08.004.

17. Kato, H.; Yamagishi, T.; Shimada, T.; Matsui, T.; Shimojima, M.; Saijo, M.; Oishi, K. Epidemiological and Clinical Features of Severe Fever with Thrombocytopenia Syndrome in Japan, 2013-2014. PLoS One 2016, 11, e0165207, http://doi.org/10.1371/journal.pone.0165207.

18. Mares-Sámano, S.; Garduño-Juárez, R. Computational modeling of the interactions of drugs with human serum albumin (HSA). Comput. y Sist. 2018, 22, 1123-1135, http://doi.org/10.13053/CyS-22-4-3085.

19. Pence, H.E.; Williams, A. ChemSpider: An Online Chemical Information Resource. J. Chem. Educ. 2010, 87, 1123-1124, http://doi.org/10.1021/ed100697w.

20. Agarwal, R.; Singh, A.; Sen, S. Role of Molecular Docking in Computer-Aided Drug Design and Development. 2016, http://doi.org/10.4018/978-1-5225-0362-0.ch001.

21. Jusko, W.J.; Gretch, M. Plasma and tissue protein binding of drugs in pharmacokinetics. Drug Metab. Rev. 1976, 5, 43-140, http://doi.org/10.3109/03602537608995839.

22. Chen, H.; Lyne, P.D.; Giordanetto, F.; Lovell, T.; Li, J. On evaluating molecular-docking methods for pose prediction and enrichment factors. J Chem Inf Model 2006, 46, 401-415, http://doi.org/10.1021/ci0503255.

23. Trott, O.; Olson, A.J. AutoDock Vina: improving the speed and accuracy of docking with a new scoring function, efficient optimization, and multithreading. J. Comput. Chem. 2010, 31, 455-461, http://doi.org/10.1002/jcc.21334.

24. Hunter, C.A.; Lawson, K.R.; Perkins, J.; Urch, C.J. Aromatic interactions. J. Chem. Soc. Perkin Trans. 2 2001, 0, 651-669, http://doi.org/10.1039/B008495F.

25. Berman, H.M.; Battistuz, T.; Bhat, T.N.; Bluhm, W.F.; Bourne, P.E.; Burkhardt, K.; Feng, Z.; Gilliland, G.L.; Iype, L.; Jain, S.; Fagan, P.; Marvin, J.; Padilla, D.; Ravichandran, V.; Schneider, B.; Thanki, N.; Weissig, H.; Westbrook, J.; Zardecki, C. The Protein Data Bank. Acta Crystallogr D Biol Crystallogr 2002, 58, 899907, http://doi.org/10.1107/s0907444902003451.

26. BIOVIA, Dassault Systèmes [Discovery Studio Modeling Environment Release 2017], San Diego: Dassault Systèmes 2016.

27. Hakmi, M.; Bouricha, E.M.; Kandoussi, I.; Harti, J.E.; Ibrahimi, A. Repurposing of known antivirals as potential inhibitors for SARS-CoV-2 main protease using molecular docking analysis. Bioinformation 2020, 16, 301-306, http://doi.org/10.6026/97320630016301.

28. Belhassan, A.; En-nahli, F.; Zaki, H.; Lakhlifi, T.; Bouachrine, M. Assessment of effective imidazole derivatives against SARS-CoV-2 main protease through computational approach. Life Sci. 2020, 118469, http://doi.org/10.1016/j.lfs.2020.118469.

29. Van Der Spoel, D.; Lindahl, E.; Hess, B.; Groenhof, G.; Mark, A.E.; Berendsen, H.J. GROMACS: fast, flexible, and free. J Comput Chem 2005, 26, 1701-1718, http://doi.org/10.1002/jcc.20291.

30. Kumari, R.; Kumar, R.; Lynn, A. g_mmpbsa--a GROMACS tool for high-throughput MM-PBSA calculations. J Chem Inf Model 2014, 54, 1951-1962, http://doi.org/10.1021/ci500020m. 